\title{
Proceso de toma de decisiones en niños y adolescentes con TDAH: Revisión sistemática
}

\author{
Josefina Rubiales ${ }^{1}$, Sebastián Urquijo ${ }^{1}$, Andrea Said ${ }^{1}$ y Guillermo Macbeth ${ }^{2}$ \\ ${ }^{1}$ Universidad Nacional de Mar del Plata, Mar del Plata, Argentina. \\ ${ }^{2}$ Universidad Nacional de Entre Ríos, Provincia de Entre Ríos, Argentina.
}

\section{Process of decision making in children and adolescents with ADHD: A systematic review}

\begin{abstract}
The objective of the present study was to perform a systematic review of studies on the process of decision making in children and adolescents diagnosed with attention deficit hyperactivity disorder (ADHD). The search process was carried out from the databases PsycInfo, MedLine, ERIC, Redalyc, Scielo and PubMed, with key words in English and Spanish and certain inclusion and exclusion criteria. A total of 20 articles were identified, which included a total of 788 children and adolescents from clinical groups and 652 from control groups. Subsequently, the studies were coded to analyze comparisons between them. The evidence presented may be useful for researchers and professionals in the field of health and education who work with children and adolescents with ADHD.
\end{abstract}

Keywords: ADHD; decision making; children; adolescents; systematic review.

Resumen: El objetivo del presente trabajo fue realizar una revisión sistemática de estudios sobre el proceso de toma de decisiones en niños y adolescentes con diagnóstico de Trastorno por déficit de atención con hiperactividad (TDAH). El proceso de búsqueda se realizó a partir de las bases de datos: PsycInfo, MedLine, ERIC, Redalyc, Scielo y PubMed, con palabras claves en inglés y español y determinados criterios de inclusión y exclusión. Se identificaron un total de 20 artículos que cumplieron con dichos criterios, lo que incluyó un total de 788 niños y adolescentes de grupos clínicos y 652 de grupos de control. Posteriormente, los estudios fueron codificados con el fin de analizar comparaciones entre los mismos. La evidencia presentada puede ser de utilidad para investigadores y profesionales del área de la salud y la educación que trabajen con niños y adolescentes con TDAH.

Palabras Claves: TDAH; toma de decisiones; niños; adolescentes; revisión sistemática.

\section{Introducción}

El Trastorno por déficit de atención con hiperactividad (TDAH) es definido como un patrón persistente de síntomas de inatención y/o hiperactividad e impulsividad que es más frecuente y grave que el observado habitualmente en las personas con un grado de desarrollo similar. La clasificación fenotípica actual del trastorno

Recibido: 12 diciembre 2016; aceptado: 1 abril 2017

Correspondencia: Josefina Rubiales, Universidad Nacional de Mar del Plata, Funes 3280, Cuerpo 5 Nivel 3, B7602AYJ, Mar del Plata, Argentina. Correo-e: josefinarubiales@gmail.com establece tres subtipos: con predominio de inatención (TDAH-I), con predominio de hiperactividad-impulsividad (TDAH-H) y combinado (TDAH-C) (APA, 2013).

Actualmente es considerado uno de los trastornos del neurodesarrollo más frecuentes en la población infantil (López-Martín, Albert, Fernández-Jaén y Carretié, 2010), con una prevalencia mundial del 5.9 a $7.1 \%$ en niños y adolescentes (Willcutt, 2012). El diagnóstico del TDAH es un proceso complejo, siendo uno de los aspectos más estudiados y controvertidos, ya que hasta el momento no se han encontrado evidencias de la existencia de marcadores neurobiológicos o de neuroimágenes aso- 
ciados de manera específica (Trujillo-Orrego, Ibáñez, y Pineda, 2012; Wolraich et al., 2005).

Los síntomas primarios del TDAH son comunes a la población general, y no es la presencia de los mismos lo que determina la disfuncionalidad, sino la intensidad y la frecuencia con la que se presentan, con respecto a la edad de desarrollo de la persona y a su contexto, y sobre todo por las consecuencias en el funcionamiento diario del individuo (Cardo y Servera, 2008). A pesar de los intentos para proporcionar descripciones más precisas de las características conductuales del TDAH, hay que reconocer que los niños con este trastorno representan una población heterogénea que presenta síntomas comunes, pero que difieren en otros aspectos importantes (Mediavilla-García, 2003). Estos síntomas pueden variar en intensidad en función de la edad y el nivel de desarrollo de la persona, disminuyendo con los años (Sánchez-Encalada y Díaz, 2009).

En este complejo escenario, el juicio clínico resulta fundamental para arribar a un diagnóstico certero y debe considerar los criterios específicos en aquellas personas en las que existen algunos síntomas de sospecha de presencia del trastorno (Catelan-Mainardes, 2010). Estos criterios se encuentran establecidos en las dos clasificaciones internacionales más destacadas: la Clasificación Internacional de Enfermedades, Organización Mundial de la Salud, CIE-10 (OMS, 1992) y el Manual diagnóstico y estadístico de los trastornos mentales de la Asociación Psiquiátrica Americana, DSM-5 (APA, 2013). Sin embargo, dichos criterios se refieren exclusivamente a las manifestaciones conductuales, y no incluyen alteraciones cognitivas, neuroanatómicas, electrofisiológicas, ni genéticas, que las investigaciones reconocen como vinculadas al TDAH (Téllez-Villagra, Valencia-Flores y Beauroyre-Hijar, 2011).

Otro aspecto a considerar es que, en la mayor parte de los casos, se presenta comorbilidad con otros síntomas asociado a otras entidades psíquicas o del comportamiento, con una frecuencia mayor a la esperada en la población general (Cardo y Amengual-Gual, 2015). Precisamente, el TDAH puro es el más infrecuente, mientras que aproximadamente en un $87 \%$ de los casos se lo encuentra asociado con otros trastornos como trastornos del aprendizaje, trastornos de ansiedad, trastornos del estado de ánimo (Ramos-Loyo, Taracena, Sánchez-Loyo, Matute, y González-Garrido, 2011). Así también puede verse asociado con el tempo cognitivo lento (Camprodon et al., 2013), y trastornos del espectro autista (García-Berjillos, Aliño, Gadea, Espert, y Salvador, 2015).

Diversas investigaciones coinciden en señalar que su sintomatología se debe a un déficit cognitivo (Barkley, 2011; Navarro y García-Villamisar, 2011). El TDAH se caracteriza por un estilo cognitivo propio y distintivo, con menor control inhibitorio y menor flexibilidad cognitiva (Barkley, 2010; Rubiales, Bakker, y Urquijo, 2013), y dificultades en organización y planificación (Barkley, 2013; Rubiales, Bakker y Delgado-Mejía, 2010), lo cual ha sido ampliamente estudiado durante las últimas dos décadas. Recientemente se han comenzado a investigar los estilos, problemas y/o déficits de las personas con TDAH en la toma de decisiones (TD), llegando a comparar esta deficiencia con la afección que genera el déficit de atención (Mowinckel, Pedersen, Eilertsen, y Biele, 2015).

Si bien aún no están claramente identificados los mecanismos cognitivos y neurobiológicos involucrados en el déficit en toma de decisiones en las personas con TDAH (Castellanos y Tannock, 2002), de acuerdo al modelo dual de Sonuga-Barke se considera que las personas con TDAH presentan una alteración en los circuitos de recompensa que provoca que la señal de recompensa demorada no sea detectada, por lo cual prefieren recompensas inmediatas, aunque pequeñas antes que recompensas mayores pero demoradas en el tiempo (Wilbertz et al., 2013). Asimismo, existen evidencias de que las personas con TDAH tienden a estar involucrados en una mayor proporción de situaciones y comportamientos de riesgo en la vida cotidiana que las personas sin TDAH (Groen, Gaastra, Lewis-Evans, y Tucha, 2013), siendo más propensas a presentar dificultades en la toma de decisiones debido a su déficit en el control de los impulsos, corriendo riesgos innecesarios sin considerar las consecuencias futuras de sus acciones (Drechsler, Rizzo, y Steinhausen, 2010; Toplak, Jain, y Tannock, 2005). Esta falla en el control de impulsos y en la toma de decisiones se manifiesta en el TDAH como un factor de riesgo para la dependencia y el abuso de sustancias como nicotina, alcohol, marihuana y cocaína, así como para el juego patológico (Lee, Humphreys, Flory, Liu, y Glass, 2011). Además, las personas con TDAH tienden a presentar un incremento en conductas de criminalidad (Mannuzza, Klein, y Moulton, 2008) y un comportamiento sexual de mayor riesgo (White y Buehler, 2012).

La TD es un proceso definido como la selección de una alternativa dentro de un rango de opciones existentes, considerando los posibles resultados de las selecciones realizadas y sus consecuencias en el comportamiento presente y futuro (Kahneman y Tversky, 1984). Diversos modelos han estudiado la TD (Kahneman, 2011), entre los cuales se encuentra el planteado por Damasio, Everitt y Bishop (1996) respecto a la Hipótesis del Marcador Somático, el cual tiene su base en las neurociencias contemporáneas. El mismo considera que la 
TD es un proceso en el que convergen los aspectos racionales y los emocionales en el análisis de cada situación, en las cuales el organismo experimentará una serie de señales emocionales denominados marcadores somáticos que actúan como alarmas emocionales al establecer vínculos con experiencias pasadas influenciando las decisiones. Éste proceso se encuentra vinculado a la región ventromedial de la corteza prefrontal (Bechara, Damasio, y Damasio, 2000).

Pueden identificarse dos tipos de toma de decisiones, el proceso de toma de decisiones bajo incertidumbre se presenta cuando no existen reglas claras para resolver el problema y la persona no tiene información sobre los resultados y la probabilidad de las consecuencias, por lo cual debe descubrir las reglas implícitamente utilizando el feedback que obtiene como resultado de sus elecciones, basándose en sus sentimientos y decidiendo intuitivamente (Bechara, 2004; Brand, Heinze, Labudda y Markowitsch, 2008). En cambio, en la toma de decisiones bajo riesgo existe información explícita sobre las consecuencias potenciales y las probabilidades de recompensas o castigos, las decisiones se realizan en base a los conocimientos disponibles sobre la situación y a las diversas opciones que se evalúan (Brand, Grabenhorst, Starcke, Vandekerckhove, y Markowitsch, 2007). Este tipo de TD está estrechamente asociada a las funciones ejecutivas (Brand et al., 2008). Esta distinción entre incertidumbre y riesgo empleada en los estudios sobre TDAH en niños se corresponde con el campo de investigación general de TD (Kahneman y Tversky, 1984; Kahneman, 2011).

A continuación, se citan y describen brevemente las pruebas de toma de decisiones bajo incertidumbre.

El Iowa Gambling Task (IGT) (Bechara, Damasio, Damasio, y Anderson, 1994) es una tarea informatizada, cuyo objetivo consiste en maximizar un capital inicial y evitar perderlo, eligiendo entre cuatro mazos de cartas, que presentan distintas ganancias y pérdidas. El Iowa Gambling Task (IGT) para niños (Garon, Moore, y Waschbusch, 2006) es una adaptación de la versión del IGT para adultos, con la misma lógica. Se trata de un juego de cartas informatizado, cuyo objetivo consiste en acumular la mayor cantidad de premios que sea posible, eligiendo entre cuatro mazos de cartas de colores.

El Hungry Donkey Task (HDT) (Crone y Van der Molen, 2004), es una prueba computarizada para niños, derivada del IGT, cuyo objetivo consiste en recolectar la mayor cantidad de manzanas verdes, seleccionando una de las cuatro puertas posibles.

El Jackpot (Luman, Oosterlaan, Knol, y Sergeant, 2008) es una tarea informatizada que presenta tres alternativas de elección (izquierda, centro o derecha), con diferentes recompensas y sanciones. Se realizan dos versiones de la prueba, una con alternativas desventajosas de mayor magnitud y otra de mayor frecuencia.

El Balloon Analogue Risk Task (BART) (Lejuez et al., 2002) requiere que el niño infle 30 globos separados, ganando dinero en función del número de veces que se bombea para inflar cada globo (a mayor número de veces que se bombea, se gana más dinero). Sin embargo, los globos pueden explotar después de un número desconocido y variable de bombeos y, en estos casos, el dinero ganado con ese globo se pierde. Existe una versión del BART en la que los participantes presionan el mouse para cada bombeo y otra en la que directamente escriben el número de bombeos para inflar cada globo sin que explote.

La Two-choice Impulsivity Task y la Five-Choice Impulsivity Task (Patros et al., 2015), son tareas informatizadas que suponen la elección de cajas (dos o cinco dependiendo de la versión) con recompensas que varían en tiempo y valor, desde pequeñas e inmediatas a grandes y diferidas.

A continuación, se citan y describen brevemente las pruebas de toma de decisiones bajo riesgo.

La Make a Match Game (Drechsler et al., 2010), es una tarea informatizada de descuento probabilístico, cuyo objetivo consiste en encontrar la copia de una carta objetivo en una línea de tarjetas con sus figuras ocultas. En cada uno de los 12 ensayos, los niños pueden elegir jugar con dos, tres o cuatro cartas con los respectivos beneficios de ganar uno, dos o tres caramelos cuando la tarjeta elegida es correcta.

El Game of Dice Task (GDT) (Brand, et al. 2005), es una tarea informatizada, cuyo objetivo consiste en aumentar el capital inicial y evitar la pérdida de dinero. El participante debe adivinar qué número aparecerá en cada uno de los tiros de un dado, y para ello puede elegir entre un único número o combinaciones de dos, tres o cuatro números. El participante gana o pierde montos diferentes en función de la cantidad de números de la opción seleccionada (un único número 100, dos números 200, tres números 500 y cuatro números 1000).

La Tarea de Descuento Temporal y la Tarea de Descuento Probabilístico (Scheres et al., 2006). En la primera los participantes son instruidos para tomar decisiones entre dos opciones, una recompensa pequeña y variable que se entrega inmediatamente y una recompensa grande y constante que se entrega después de una demora variable. En la segunda los participantes deben tomar decisiones entre una recompensa pequeña que se entrega con $100 \%$ de certeza y una recompensa más grande que se entrega con una probabilidad entre 0 y 1 .

La Cambridge Gamble Task (Rogers et al., 1999), es una prueba computarizada en la que se presenta una lí- 
nea de diez cuadros rojos y azules en una pantalla y el número de cuadros de cada color varía en cada ensayo. El objetivo consiste en adivinar qué color presentará un cuadro que se muestra resaltado y, a continuación, apostar un porcentaje de los puntos totales, que puede ser ganado o perdido.

Estudios sobre los procesos de TD realizados con el WISC-III en niños con TDAH han aportado evidencias de los beneficios de utilizar estímulos auditivos en las consignas y de dificultades con los procesos de estimación de costo y beneficio, por lo que proponen que el mencionado test podría llegar a utilizarse como instrumento para la evaluación de la TD en niños con TDAH (Yasumura, Takimoto, Nakazawa, y Inagaki, 2016).

Diversos estudios investigaron el desempeño de niños y adolescentes con TDAH en tareas de TD y informaron resultados inconsistentes, sin evidencias concluyentes, mostrando la mitad de los estudios que la TD es más arriesgada en esta población, independientemente del tipo de tarea utilizada (Groen et al., 2013), y no se encontraron estudios de comparación de ambos tipos de toma de decisiones. De acuerdo a Ziegler, Pedersen, Mowinckel, y Biele (2016) la ausencia de evidencias confirmatorias de los modelos computacionales de los procesos de TD en niños con TDAH, probablemente se deba a problemas generados por estudios clínicos con pequeñas muestras de conveniencia que podrían no ser lo suficientemente grandes para detectar efectos robustos pero pequeños.

Abordar el estudio del neurodesarrollo en la infancia y la adolescencia y los déficits que puedan presentarse resulta fundamental para la comprensión del funcionamiento cognitivo y su posterior aplicación al diseño de currículos educativos e intervenciones en salud (Verdejo-García y Tirapu-Ustárroz, 2012). La evaluación temprana del desarrollo de la TD en niños y adolescentes con TDAH podría ser útil en la identificación de dificultades, aportando información relevante a la conceptualización del trastorno para profundizar el modelo de evaluación y diseñar tratamientos acordes a las necesidades particulares de esta población, que permitan mejorar su calidad de vida. Por este motivo se propuso realizar una revisión sistemática del estado de los conocimientos derivados de investigaciones y citados en la literatura científica en los últimos diez años sobre los procesos de TD en niños y adolescentes con TDAH y los instrumentos de evaluación utilizados. La revisión sistemática de la evidencia científica es una herramienta metodológica de investigación que ofrece la posibilidad de informar, actualizar e integrar de forma eficiente la información disponible acerca de una temática específica, utilizando métodos sistemáticos y explícitos para identificar, selec- cionar y valorar críticamente las investigaciones relevantes (Perestelo-Pérez, 2013; Sánchez-Meca y Botella-Ausina, 2010).

\section{Método}

El proceso de revisión bibliográfica consistió en la búsqueda de estudios en las bases de datos PsycInfo, MedLine, ERIC, Redalyc, Scielo y PubMed. Dicha búsqueda fue efectuada en enero de 2016, utilizando las palabras claves en inglés: decision-making, gambling task, attention deficit hyperactivity disorder, ADHD, children, adolescents; en español: toma de decisiones, prueba de azar, Trastorno por déficit de atención e hiperactividad, TDAH, niños, adolescentes; y en portugués: tomada de decisão, teste aleatório, transtorno de déficit de atenção e hiperactividade, TDAH, crianças, adolescentes. Una vez que fueron detectados los artículos, fueron seleccionados para la revisión aquellos que cumplían con los siguientes criterios: (1) haber sido publicados entre los años 2005 y 2016; (2) estar escritos en idioma inglés, español o portugués; (3) incluir grupo experimental con diagnóstico de TDAH y grupo control, descartando los diseños $\mathrm{N}=1$; (4) evaluar el proceso de toma de decisiones en las muestras estudiadas; y (5) abordar una población de niños y adolescentes con edades de 6 a 18 años.

El proceso de búsqueda tuvo como resultado inicial 256 referencias que fueron analizadas por los revisores, de forma independiente, seleccionando en función de los títulos y la información de los resúmenes a aquellas potencialmente relevantes según los criterios de inclusión. Se seleccionaron 26 publicaciones para un análisis crítico, y posteriormente se repitió el análisis de los artículos completos y se incluyeron en el estudio 20 artículos que cumplieron estrictamente con los criterios de selección, aportando información sobre un total de 26 grupos clínicos y 21 grupos control. El tamaño muestral promedio fue de 30,6 integrantes, con una muestra total de 788 niños y adolescentes de grupos clínicos y 652 de grupos de control.

\section{Proceso de codificación de los estudios}

Se incluyeron las siguientes variables de los participantes: (a) edad promedio en años; (b) desviación típica (DT) de la edad promedio; (c) porcentaje de varones de cada muestra; (d) promedio de CI (coeficiente intelectual); (e) DT del promedio de CI; (f) análisis de existencia de comorbilidad; (g) análisis del subtipo; (h) criterios diagnósticos utilizados; (i) exposición a tratamiento. En cuanto a las características metodológicas de las in- 


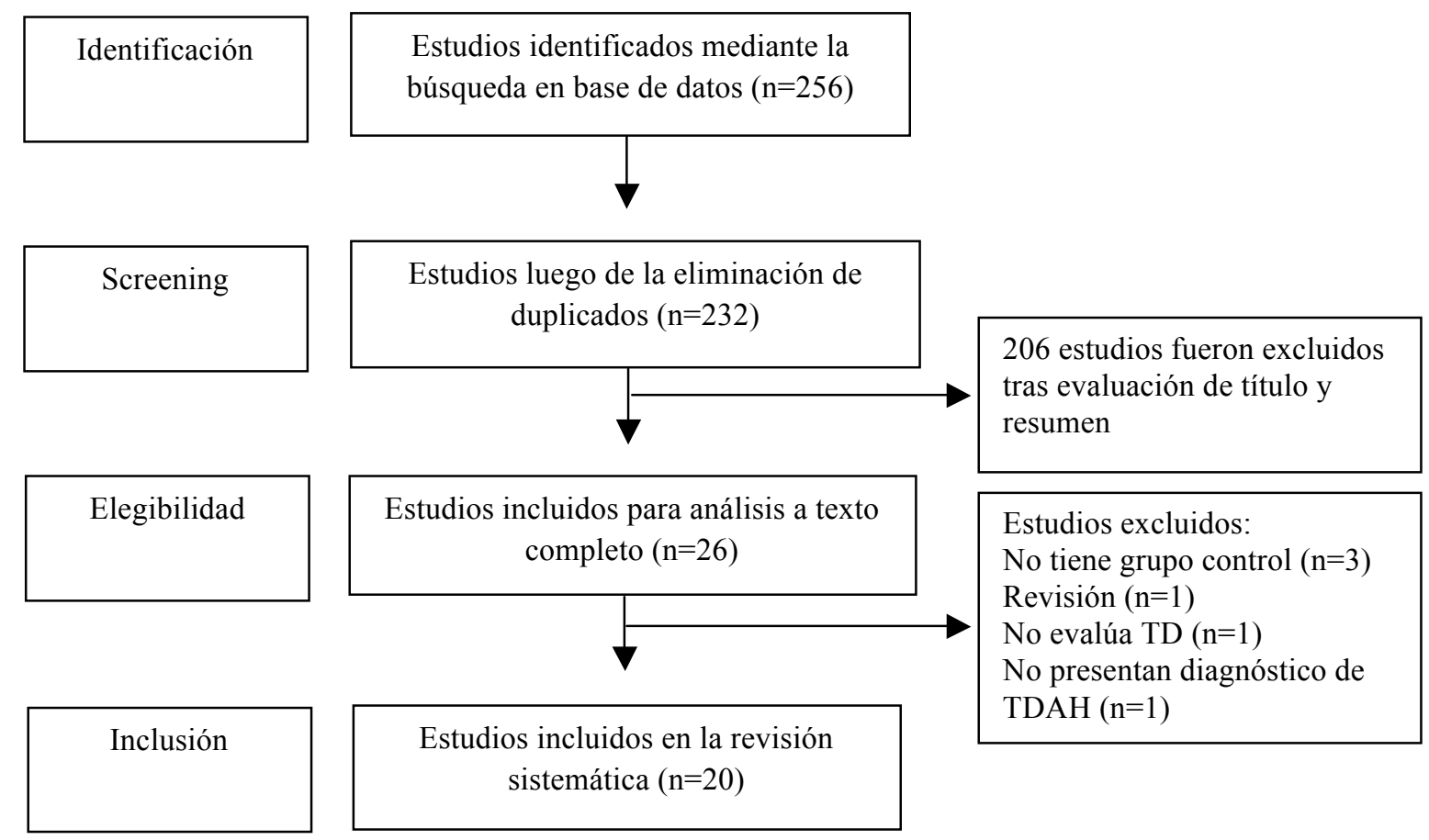

Figura 1. Diagrama de flujo para la identificación de los estudios.

vestigaciones, se codificaron: (a) N del grupo clínico; (b) $\mathrm{N}$ del grupo control; (c) instrumento (prueba o tarea) de toma de decisiones; (d) cantidad de ensayos de la prueba; (e) cantidad de opciones de la prueba; (f) características de las opciones; (g) tipo de recompensa; $(\mathrm{h})$ medida de salida del resultado; (i) media (M) del grupo control en la prueba de toma de decisiones; (j) DT de la $M$ del grupo control en la prueba de toma de decisiones; (k) $M$ del grupo clínico en la prueba de toma de decisiones; (1) $D T$ de la $\mathrm{M}$ del grupo clínico en la prueba de toma de decisiones. Y, por último, se consideraron el año de publicación del estudio y el país donde se realizó el estudio.

La codificación de algunas de las variables requirió la adopción de juicios de decisión, para lo cual se realizó un estudio de la fiabilidad del proceso de codificación. El método implementado consistió en que dos investigadores codificaron de forma independiente tanto las variables nominales como las variables numéricas de una muestra aleatoria del $20 \%$ del total de los estudios seleccionados. Se establecieron las semejanzas y diferencias entre ambas codificaciones para lo cual se puntuaron en forma numérica cada una de ellas, situándose el grado de acuerdo (cantidad de variables codificadas de modo coincidente) en torno al $90 \%$ en todas las variables codificadas, lo que resulta altamente satisfactorio (Orwin, 1994). Las inconsistencias entre los codificadores se resolvieron por consenso.

\section{Análisis estadístico}

Para realizar los análisis estadísticos se utilizó el paquete estadístico SPSS, versión 19. Con el objeto de describir el desempeño de la TD en niños y adolescentes con TDAH en cada uno de los estudios, se realizaron análisis estadístico descriptivo de $M$ y $D T$. Asimismo, con el propósito de establecer si las diferencias observadas son estadísticamente significativas, se calculó el tamaño del efecto de las diferencias utilizando la clasificación por rangos de Cohen, el cual se obtuvo directamente a partir de las M y DT y fue clasificado como grande (mayor a .8), mediano (cercano a .5) o pequeño (menor a .2) (Cohen, 1988; Quezada, 2007). No todos los estudios presentaron datos suficientes sobre el tamaño del efecto o las $M$ y $D T$ para su cálculo.

\section{Resultados}

Los 20 artículos seleccionados para la presente revisión se presentan en la Tabla 1. Los mismos estaban escritos en idioma inglés y procedían de países diferentes. Se encontró que un $20 \%$ de los estudios fueron realizados en Estados Unidos, seguidos por un 15\% en Noruega, mientras que el resto de las investigaciones fueron realizadas en Canadá (10\%), Suiza (10\%), Países Bajos $(10 \%)$, Israel (10\%), Inglaterra (10\%), Dinamarca (5\%), Brasil (5\%) y Japón (5\%). Para realizar el diagnóstico de 
Tabla 1. Variables individuales de cada estudio de la revisión.

\begin{tabular}{|c|c|c|c|c|c|c|c|}
\hline \multirow[b]{2}{*}{ Estudio } & \multirow[b]{2}{*}{ Grupo de edad } & \multicolumn{3}{|c|}{ Grupo clínico } & \multicolumn{3}{|c|}{ Grupo control } \\
\hline & & $\begin{array}{c}\mathrm{N} \\
(\% \text { varones })\end{array}$ & $\begin{array}{l}\text { M edad } \\
\quad(D T)\end{array}$ & $\begin{array}{l}M \mathrm{CI} \\
(D T)\end{array}$ & $\begin{array}{c}\mathrm{N} \\
(\% \text { varones })\end{array}$ & $\begin{array}{l}M \text { edad } \\
\quad(D T)\end{array}$ & $\begin{array}{l}M \mathrm{CI} \\
(D T)\end{array}$ \\
\hline 1. Toplak et al. (2005) & $\begin{array}{c}\text { Adolescentes } \\
13 \text { a } 18\end{array}$ & $44(\mathrm{~S} / \mathrm{D})$ & $15.6(1.4)$ & $\begin{array}{l}104.1 \\
(10.1)\end{array}$ & $34(\mathrm{~S} / \mathrm{D})$ & $15.4(1.5)$ & $\begin{array}{l}109.4 \\
(10.2)\end{array}$ \\
\hline $\begin{array}{l}\text { 2. Garon, Moore, \& } \\
\text { Waschbusch (2006) }\end{array}$ & $\begin{array}{l}\text { Niños } \\
6 \text { a } 13\end{array}$ & $\begin{array}{c}\text { TDAH: } 11 \\
\text { TDAH+Ans-Depr: } 10 \\
(80.95 \%)\end{array}$ & $9.86(1.71)$ & $\begin{array}{c}95.76 \\
(17.18)\end{array}$ & $21(80.95 \%)$ & $9.81(1.94)$ & $\begin{array}{c}104.8 \\
(13.66)\end{array}$ \\
\hline $\begin{array}{l}\text { 3. Geurts, Van der Oord, } \\
\text { \& Crone (2006) }\end{array}$ & $\begin{array}{l}\text { Niños } \\
8 \text { a } 12\end{array}$ & $20(85 \%)$ & $9.9(1.1)$ & $\begin{array}{l}106.9 \\
(15.4)\end{array}$ & $22(81.81 \%)$ & $10(1.3)$ & $\begin{array}{l}108.9 \\
(16.2)\end{array}$ \\
\hline $\begin{array}{l}\text { 4. Scheres, et al. } \\
(2006)\end{array}$ & $\begin{array}{c}\text { Niños y } \\
\text { adolescentes } \\
6 \text { a } 17\end{array}$ & $\begin{array}{c}\text { 6-11 años: } 11 \\
\text { 12-17 años: } \\
11 \\
(73.91 \%)\end{array}$ & $11.6(1.6)$ & $\begin{array}{l}105.65 \\
(15.85)\end{array}$ & $24(70.83 \%)$ & $11.7(1.6)$ & $\begin{array}{l}101.6 \\
(13.1)\end{array}$ \\
\hline 5. DeVito et al. (2008) & Niños 7 a 13 & $21(100 \%)$ & $10(2.05)$ & $\mathrm{S} / \mathrm{D}$ & $22(100 \%)$ & $\begin{array}{l}10.32 \\
(1.59)\end{array}$ & $\mathrm{S} / \mathrm{D}$ \\
\hline $\begin{array}{l}\text { 6. Drechsler et al. } \\
(2008)\end{array}$ & $\begin{array}{l}\text { Niños } \\
11 \text { a } 13\end{array}$ & $23(91.3 \%)$ & $12.2(0.8)$ & $\begin{array}{l}101.3 \\
(10.7)\end{array}$ & $24(95.83 \%)$ & $11.9(0.6)$ & $\begin{array}{l}108.5 \\
(18.5)\end{array}$ \\
\hline $\begin{array}{l}\text { 7. Luman, Oosterlaan, } \\
\text { Knol, \& Sergeant } \\
(2008)\end{array}$ & $\begin{array}{l}\text { Niños } \\
7 \text { a } 12\end{array}$ & $23(78.26 \%)$ & $9.65(1.41)$ & $\begin{array}{c}98.9 \\
(11.3)\end{array}$ & $20(75 \%)$ & $9.43(1.37)$ & $\begin{array}{l}114.7 \\
(14.3)\end{array}$ \\
\hline $\begin{array}{l}\text { 8. Masunami, Okazaki } \\
\text { \& Maekawa (2009) }\end{array}$ & $\begin{array}{c}\text { Niños y } \\
\text { adolescentes } 7 \text { a } \\
14\end{array}$ & $14(92.5 \%)$ & $11.5(2.2)$ & $\begin{array}{l}102.2 \\
(12.2)\end{array}$ & $11(54.54 \%)$ & $11.7(1.7)$ & $\mathrm{S} / \mathrm{D}$ \\
\hline $\begin{array}{l}\text { 9. Drechsler et al. } \\
\text { (2010) }\end{array}$ & $\begin{array}{l}\text { Niños } \\
7 \text { a } 10\end{array}$ & $28(71.42 \%)$ & $9.2(1,2)$ & $\begin{array}{l}106.3 \\
(15.5)\end{array}$ & $28(71.42 \%)$ & $9.2(1.4)$ & $\begin{array}{l}108.2 \\
(13.9)\end{array}$ \\
\hline $\begin{array}{l}\text { 10. Lambek, et al. } \\
\text { (2011). }\end{array}$ & $\begin{array}{c}\text { Niños y } \\
\text { adolescentes } \\
7 \text { a } 14\end{array}$ & $\begin{array}{c}\text { TDAH: } 22 \\
(86 \%) \\
\text { TDAH+TFE: } \\
26(73 \%)\end{array}$ & $\begin{array}{c}\text { TDAH: } \\
10.32 \\
(1.67) \\
\text { TDAH+T- } \\
\text { FE: } 10.62 \\
(2.00)\end{array}$ & $\begin{array}{c}\text { TDAH: } \\
94.77 \\
(13.32) \\
\text { TDAH+T- } \\
\text { FE: } 85.9 \\
(10.83)\end{array}$ & $26(77 \%)$ & $10.77(1.7)$ & $\begin{array}{l}106.85 \\
(14.05)\end{array}$ \\
\hline $\begin{array}{l}\text { 11. Hobson, Scott \& } \\
\text { Rubia (2011) }\end{array}$ & $\begin{array}{c}\text { Niños y } \\
\text { adolescentes } 10 \\
\text { a } 17\end{array}$ & $31(83.87 \%)$ & $\begin{array}{l}13.32 \\
(1.81)\end{array}$ & $\begin{array}{c}95.52 \\
(13.06)\end{array}$ & $34(73.53 \%)$ & $\begin{array}{l}13.13 \\
(1.99)\end{array}$ & $\begin{array}{c}106.5 \\
(12.12)\end{array}$ \\
\hline $\begin{array}{l}\text { 12. Humphreys, \& Lee } \\
\text { (2011) }\end{array}$ & $\begin{array}{l}\text { Niños } \\
5 \text { a } 10\end{array}$ & $103(77 \%)$ & $7.3(1.11)$ & $\mathrm{S} / \mathrm{D}$ & $100(66 \%)$ & $7.49(1.12)$ & $\mathrm{S} / \mathrm{D}$ \\
\hline $\begin{array}{l}\text { 13. Sallum, Mata, } \\
\text { Miranda, \& } \\
\text { Malloy-Diniz (2013) }\end{array}$ & $\begin{array}{c}\text { Niños y } \\
\text { adolescentes } \\
7 \text { a } 14\end{array}$ & $24(75 \%)$ & $\begin{array}{l}10.04 \\
(1.65)\end{array}$ & $\mathrm{S} / \mathrm{D}$ & $24(62.5 \%)$ & $\begin{array}{l}10.29 \\
(1.54)\end{array}$ & $\mathrm{S} / \mathrm{D}$ \\
\hline $\begin{array}{l}\text { 14. Skogli, Egeland, } \\
\text { Andersen, Hovik, \& } \\
\text { Oie (2013) }\end{array}$ & $\begin{array}{c}\text { Niños y } \\
\text { adolescentes } \\
8 \text { a } 17\end{array}$ & $\begin{array}{l}\text { TDAH-C: } 36 \\
(63.88 \%) \\
\text { TDAH-I: } 44 \\
(45.45 \%)\end{array}$ & $\begin{array}{c}\text { TDAH-C: } \\
11.84 \\
(2.15) \\
\text { TDAH-I: } \\
12.11 \\
(1.95)\end{array}$ & $\begin{array}{c}\text { TDAH-C: } \\
96.6 \\
(13.3) \\
\text { TDAH-I: } \\
94.2(15)\end{array}$ & $50(64 \%)$ & $12(2.03)$ & $\begin{array}{l}103.8 \\
(12.9)\end{array}$ \\
\hline $\begin{array}{l}\text { 15. Kroyzer, Gross-Tsur, } \\
\text { \& Pollak (2014) }\end{array}$ & $\begin{array}{l}\text { Adolescentes } \\
13 \text { a } 18\end{array}$ & $32(62.5 \%)$ & $15.5(1.4)$ & $113.9(16)$ & $32(65.62 \%)$ & $15.9(1.8)$ & $\begin{array}{l}114.4 \\
(16.2)\end{array}$ \\
\hline $\begin{array}{l}\text { 16. Skogli, Andersen, } \\
\text { Hovik, \& Oie (2014) }\end{array}$ & $\begin{array}{c}\text { Niños y } \\
\text { adolescentes } \\
9 \text { a } 16\end{array}$ & $75(52 \%)$ & $11.6(1.95)$ & $\begin{array}{l}95.45 \\
(14.6)\end{array}$ & $47(61.7 \%)$ & $11.5(1.9)$ & $\begin{array}{c}104.8 \\
(13.05)\end{array}$ \\
\hline
\end{tabular}


Tabla 1. Variables individuales de cada estudio de la revisión (Continuación)

\begin{tabular}{|c|c|c|c|c|c|c|c|}
\hline \multirow[b]{2}{*}{ Estudio } & \multirow[b]{2}{*}{ Grupo de edad } & \multicolumn{3}{|c|}{ Grupo clínico } & \multicolumn{3}{|c|}{ Grupo control } \\
\hline & & $\begin{array}{c}\mathrm{N} \\
(\% \text { varones })\end{array}$ & $\begin{array}{l}\text { M edad } \\
\quad(D T)\end{array}$ & $\begin{array}{l}M \mathrm{CI} \\
(D T)\end{array}$ & $\begin{array}{c}\mathrm{N} \\
(\% \text { varones })\end{array}$ & $\begin{array}{l}M \text { edad } \\
\quad(D T)\end{array}$ & $\begin{array}{l}M \mathrm{CI} \\
(D T)\end{array}$ \\
\hline $\begin{array}{l}\text { 17. Antonini, , et al. } \\
\text { (2015). }\end{array}$ & $\begin{array}{l}\text { Niños } \\
7 \text { a } 12\end{array}$ & $\begin{array}{c}\text { TDAH: } 67 \\
(75.76 \%) \\
\text { TDAH+TND: } \\
33(72.72 \%)\end{array}$ & $\begin{array}{c}\text { TDAH: } \\
8.88(1.48) \\
\text { TDAH+ } \\
\text { TND: } 9.44 \\
(1.75)\end{array}$ & $\begin{array}{c}\text { TDAH: } \\
104.51 \\
(12.03) \\
\text { TDAH+ } \\
\text { TND: } \\
101.88 \\
(12.67)\end{array}$ & $30(66.67 \%)$ & $9(1.8)$ & $\begin{array}{l}116.77 \\
(10.47)\end{array}$ \\
\hline 18. Hovik, et al. (2015) & $\begin{array}{c}\text { Niños y } \\
\text { adolescentes } \\
10-16\end{array}$ & $33(60.6 \%)$ & $12(2.16)$ & $97(14)$ & $50(64 \%)$ & $12(2)$ & $104(13)$ \\
\hline 19. Patros et al. (2015). & $\begin{array}{l}\text { Niños } \\
8 \text { a } 12\end{array}$ & $16(100 \%)$ & $9.71(1.63)$ & $\begin{array}{l}98.13 \\
(8.96)\end{array}$ & $23(100 \%)$ & $10(1.44)$ & $\begin{array}{l}108.39 \\
(13.09)\end{array}$ \\
\hline $\begin{array}{l}\text { 20. Pollak, \& Shoham } \\
\text { (2015). }\end{array}$ & $\begin{array}{c}\text { Adolescentes } \\
13 \text { a } 18\end{array}$ & $30(66.66 \%)$ & $14.9(1.5)$ & $\begin{array}{l}105.1 \\
(10.1)\end{array}$ & $30(30 \%)$ & $15.2(1.9)$ & $\begin{array}{l}108.9 \\
(14.5)\end{array}$ \\
\hline
\end{tabular}

Nota: S/D: Sin dato. TDAH+Ans-Depr: TDAH + ansiedad-depresión. TFE: trastorno de funciones ejecutivas. TDAH+TND: TDAH + trastorno negativista desafiante.

TDAH han sido en su mayoría los criterios del DSM IV y, en algunos casos, el CIE-10. En la Tabla 1 se presentan los datos sobre los sujetos incluidos en cada uno de los estudios.

Como puede observarse en la Tabla 1, la totalidad de los estudios incluyeron información sobre el número de sujetos y los grupos de edad incluidos en la muestra. Sólo uno no incluye información sobre el sexo de los participantes. En cuanto a la población de estudio, el $45 \%$ de las investigaciones (9) se realizaron solo con niños (hasta 13 años), el 40\% de los estudios (8) fueron con niños y adolescentes, y el 15\% restante (3) sólo con adolescentes ( 3 investigaciones con jóvenes de 13 a 18 años). Respecto a las fechas de publicación, el 50\% de los artículos fueron publicados en el periodo 2005-2010 frente al 50\% publicado entre 2011 y 2015 . Respecto al género, se observa que todos los estudios evaluaron mayor cantidad de varones que de mujeres, lo cual se vincula con lo informado en el DSM-5 respecto a una mayor presencia del sexo masculino (APA, 2013).

En la Tabla 2 se presentan los instrumentos que fueron utilizados en cada uno de los estudios, el tipo de TD que evalúa (bajo incertidumbre o bajo riesgo), la cantidad de ensayos, el número de opciones para elegir y el tipo de recompensa, con el objetivo de analizar en detalle cada uno de los instrumentos utilizados para evaluar toma de decisiones.

Como puede observarse, los 20 estudios analizados utilizaron nueve instrumentos para evaluar la TD en niños y adolescentes. La mayoría de los estudios (70\%) utiliza tareas de TD bajo incertidumbre, mientras que el $30 \%$ restante utiliza tareas de TD bajo riesgo. Respecto a la cantidad de ensayos, la media es de 96.10, con un valor mínimo de 12 y un máximo de 200 ensayos. En cuanto a la cantidad de opciones, la media es de 4.14, con un mínimo de dos y un máximo de diez opciones. Y respecto al tipo de recompensas, el 65\% utilizó premios ficticios como dinero, puntos o manzanas, mientras que el restante $35 \%$ utilizó premios reales, como caramelos o dinero.

En la Tabla 3 se presentan los estadísticos descriptivos de los resultados de las tareas, grupos clínicos y control y la d de Cohen de los 11 estudios (55\%) de los 20 analizados, que informan haber observado diferencias significativas entre los sujetos con TDAH y los grupos de control.

Como se observa en la Tabla 3, estos estudios encontraron diferencias significativas entre los niños y adolescentes de los grupos clínico y control, en todos los casos con desempeños inferiores (mayor riesgo o elecciones desventajosas) en los niños con diagnóstico de TDAH. Algunos estudios (12 y 13) no presentaron datos precisos de las medias y los DE, sin embargo, describieron sus resultados de modo tal que pudieron ser considerados para su inclusión en el presente estudio.

Del total de los estudios descriptos en la Tabla 3 (11), cuatro $(36 \%)$ analizaron la TD bajo riesgo y siete $(64 \%)$ la TD bajo incertidumbre. Entre los estudios que analizaron la TD bajo riesgo, Drechsler, Rizzo y Steinhausen (2008) no observaron diferencias significativas entre el 
Tabla 2. Características de instrumentos para la evaluación toma de decisiones en niños y adolescentes

\begin{tabular}{|c|c|c|c|c|c|}
\hline Prueba de TD & Autor & Tipo de TD & $\begin{array}{l}\text { Cantidad } \\
\text { de ensayos }\end{array}$ & $\begin{array}{l}\text { Opciones para } \\
\text { elegir }\end{array}$ & Recompensa \\
\hline 1. IGT & Bechara et al., (1994) & Incertidumbre & 100 & 4 & $\begin{array}{l}\text { Recompensas } \\
\text { ficticias }\end{array}$ \\
\hline 2. IGT para niños & Garon, et al. (2006) & Incertidumbre & 80 & 4 & $\begin{array}{l}\text { Caramelos } \\
\text { reales }\end{array}$ \\
\hline 3. HDT,versión estandar e inversa. & $\begin{array}{l}\text { Crone \& Van der } \\
\text { Molen (2004) }\end{array}$ & Incertidumbre & 200 cada versión & 4 & $\begin{array}{l}\text { Manzanas } \\
\text { ficticias }\end{array}$ \\
\hline $\begin{array}{l}\text { 4. 1. Tarea descuento temporal. } \\
\text { 2. Tarea descuento probabilístico }\end{array}$ & Scheres et al. (2006) & Riesgo & $\begin{array}{l}1: 60 \\
2: 120\end{array}$ & $\begin{array}{l}1: 2 \\
2: 2\end{array}$ & Dinero real \\
\hline 5. CGT & Rogers (1999) & Riesgo & $\mathrm{S} / \mathrm{D}$ & 2 & $\begin{array}{l}\text { Puntos } \\
\text { ficticios }\end{array}$ \\
\hline 6. GDT & Brand et al., (2005) & Riesgo & $\begin{array}{l}1^{\circ} \text { intento: } 18 \\
2^{\circ} \text { intento: } 12\end{array}$ & 4 & Dinero ficticio \\
\hline 7. Jackpot & Luman et al. 2008 & Incertidumbre & 180 & 3 & Dinero real \\
\hline 8. IGT para niños & Garon et al. (2006) & Incertidumbre & 100 & 4 & $\begin{array}{l}\text { Puntos } \\
\text { ficticios }\end{array}$ \\
\hline 9. Make-a-Match Game & $\begin{array}{l}\text { Drechsler et al. } \\
(2010) \text {. }\end{array}$ & Riesgo & 12 & 3 & $\begin{array}{l}\text { Caramelos } \\
\text { reales }\end{array}$ \\
\hline 10. HDT & $\begin{array}{l}\text { Crone \& Van der } \\
\text { Molen (2004) }\end{array}$ & Incertidumbre & 200 & 4 & $\begin{array}{l}\text { Manzanas } \\
\text { ficticias }\end{array}$ \\
\hline 11. IGT & Bechara et al., (1994) & Incertidumbre & $\mathrm{S} / \mathrm{D}$ & 4 & Dinero real \\
\hline 12. BART & Lejuez et al. (2002) & Incertidumbre & 30 & Variable & Dinero ficticio \\
\hline 13. IGT & Bechara et al., (1994) & Incertidumbre & 100 & 4 & Dinero ficticio \\
\hline 14. HDT & $\begin{array}{l}\text { Crone \& Van der } \\
\text { Molen (2004) }\end{array}$ & Incertidumbre & 150 (en 10 bloques) & 4 & $\begin{array}{l}\text { Manzanas } \\
\text { ficticias }\end{array}$ \\
\hline 15. CGT, versión modificada & Kroyzer et al. (2014) & Riesgo & 42 (en 3 rondas) & $\begin{array}{l}\text { Apuestas: } 10 \\
(10 \text { a } 100) \\
\text { Cajas para } \\
\text { elegir entre } 2 \\
\text { colores: } 10\end{array}$ & $\begin{array}{l}\text { Puntos } \\
\text { ficticios }\end{array}$ \\
\hline 16. HDT & $\begin{array}{l}\text { Crone \& Van der } \\
\text { Molen (2004) }\end{array}$ & Incertidumbre & 150 (en 10 bloques) & 4 & $\begin{array}{l}\text { Manzanas } \\
\text { ficticias }\end{array}$ \\
\hline 17. IGT para niños & Garon et al. (2006) & Incertidumbre & 80 & 4 & $\begin{array}{l}\text { Objetos } \\
\text { ficticios }\end{array}$ \\
\hline 18. HDT & $\begin{array}{l}\text { Crone \& Van der } \\
\text { Molen (2004) }\end{array}$ & Incertidumbre & 150 & 4 & $\begin{array}{l}\text { Manzanas } \\
\text { ficticias }\end{array}$ \\
\hline $\begin{array}{l}\text { 19. 1.Two-choice impulsivity task } \\
\text { 2.Five-choice impulsivity task }\end{array}$ & Patros et al. (2015) & Incertidumbre & 10 minutos & $\begin{array}{l}1.2 \\
2.5\end{array}$ & Premio real \\
\hline 20. CGT, versión modificada & $\begin{array}{l}\text { Versión modificada } \\
\text { de Rogers (1999) }\end{array}$ & Riesgo & 42 (en 3 rondas) & $\begin{array}{l}\text { Apuestas: } 10 \\
\text { Cajas para } \\
\text { elegir entre } 2 \\
\text { colores: } 10\end{array}$ & Dinero real \\
\hline
\end{tabular}

Nota: S/D: sin dato. BART: The Balloon Analogue Risk Task. CGT: Cambridge Gamble Task. GDT: Game of Dice Task. HDT: Hungry Donkey Task. IGT: Iowa Gambling Task 
Tabla 3. Resultados de los estudios que obtuvieron diferencias significativas

\begin{tabular}{|c|c|c|c|c|c|c|}
\hline Estudio & Prueba de TD & Medida de salida & $\begin{array}{c}\text { M grupo } \\
\text { control }(D T)\end{array}$ & $\begin{array}{c}\text { M grupo } \\
\text { clínico }(D T)\end{array}$ & $d$ de Cohen & Resumen de resultados \\
\hline \multirow[t]{4}{*}{1.} & IGT & Puerta A & $24.4(7.3)$ & $23.3(9.7)$ & 0.13 & \multirow{4}{*}{$\begin{array}{l}\text { TDAH desempeño }<\text { que GC } \\
\text { TDAH-C=TDAH-I }\end{array}$} \\
\hline & & Puerta B & $29.4(10.4)$ & $34.1(10)$ & 0.46 & \\
\hline & & Puerta C & $20.3(8.8)$ & $19.9(8.3)$ & 0.05 & \\
\hline & & Puerta D & $26.9(12.2)$ & $21.6(7)$ & 0.53 & \\
\hline 2. & IGT niños & Elecciones ventajosas & $\mathrm{S} / \mathrm{D}$ & $\mathrm{S} / \mathrm{D}$ & 1.14 & $\begin{array}{l}\text { TDAH desempeño }<\text { que } \\
\text { TDAH+AD y que GC }\end{array}$ \\
\hline \multirow[t]{2}{*}{6.} & GDT & $\begin{array}{l}\text { Elecciones de riesgo } \\
\text { Juego } 1\end{array}$ & $6.1(3.7)$ & $6.3(4.3)$ & 0.05 & \multirow[t]{2}{*}{ TDAH desempeño $<$ que GC } \\
\hline & & $\begin{array}{l}\text { Elecciones de riesgo } \\
\text { Juego } 2\end{array}$ & $2.9(2.8)$ & $5.60(3.7)$ & 0.83 & \\
\hline 7. & Jackpot & $\begin{array}{l}\% \text { de elecciones } \\
\text { ventajosas y } \\
\text { desventajosas }\end{array}$ & $\mathrm{S} / \mathrm{D}$ & $\mathrm{S} / \mathrm{D}$ & $\mathrm{S} / \mathrm{D}$ & $\begin{array}{l}\text { Frecuencia: TDAH=GC } \\
\text { Magnitud: TDAH desempeño } \\
<\text { que GC }\end{array}$ \\
\hline \multirow[t]{4}{*}{9.} & $\begin{array}{l}\text { Make-a-Match } \\
\text { Game }\end{array}$ & $\begin{array}{l}\text { Elección de } 2 \\
\text { probabilidades }\end{array}$ & $4.61(2.6)$ & $3.21(1.8)$ & 0.62 & \multirow[t]{4}{*}{ TDAH desempeño $<$ que GC } \\
\hline & & $\begin{array}{l}\text { Elección de } 3 \\
\text { probabilidades }\end{array}$ & $4.11(2)$ & $3.61(2.1)$ & 0.24 & \\
\hline & & $\begin{array}{l}\text { Elección de } 4 \\
\text { probabilidades }\end{array}$ & $3.25(1.8)$ & $4.89(2.5)$ & 0.75 & \\
\hline & & $\begin{array}{l}\text { Cantidad de caramelos } \\
\text { ganados }\end{array}$ & $6.32(2.4)$ & $6.21(2.8)$ & 0.04 & \\
\hline 11. & IGT & $\begin{array}{l}\text { Decisiones arriesgadas } \\
\text { (A y B) }\end{array}$ & $21.59(9.44)$ & $27.51(7.51)$ & 0.69 & TDAH desempeño $<$ que GC \\
\hline 12. & BART & $\begin{array}{l}\text { Sensibilidad al castigo } \\
\text { (restar el número de } \\
\text { bombas del ensayo } \\
\text { posterior a que un globo } \\
\text { explote a partir del número } \\
\text { de bombas precedente a } \\
\text { que un globo explote). }\end{array}$ & $\mathrm{S} / \mathrm{D}$ & $\mathrm{S} / \mathrm{D}$ & $\mathrm{S} / \mathrm{D}$ & $\begin{array}{l}\text { TDAH+ TND }>\text { conductas de } \\
\text { riesgo que los TDAH y estos } \\
>\text { conductas de riesgo que los } \\
\text { controles. }\end{array}$ \\
\hline 13. & IGT & $\begin{array}{l}\mathrm{N}^{\circ} \text { de mazos desventajosos } \\
\text { - } \mathrm{N}^{\circ} \text { de mazos ventajosas y } \\
\text { la cantidad de estrategias } \\
\text { de mantenerse y cambiar la } \\
\text { elección }\end{array}$ & $\mathrm{S} / \mathrm{D}$ & $\mathrm{S} / \mathrm{D}$ & $\mathrm{S} / \mathrm{D}$ & $\begin{array}{l}\text { TDAH=GC en los puntajes } \\
\text { estándar y TDAH diferente en } \\
\text { las estrategias elegidas de } \\
\text { cambio de puertas. }\end{array}$ \\
\hline 15. & $\begin{array}{l}\text { CGT, versión } \\
\text { modificada }\end{array}$ & Suma de la apuesta & $64.18(15.44)$ & $55.96(13.82)$ & 0.56 & TDAH desempeño $<$ que GC \\
\hline 19. & $\begin{array}{l}\text { 1.Two-choice } \\
\text { impulsivity task } \\
\text { 2.Five-choice } \\
\text { impulsivity task }\end{array}$ & Total de puntos & $\begin{array}{l}2 \text { opciones: } \\
361.43(25.88) \\
5 \text { opciones: } \\
202.91(22.81)\end{array}$ & $\begin{array}{l}2 \text { op: } 335.63 \\
(25.5) \\
5 \text { op: } 196.88 \\
(28.88)\end{array}$ & $\begin{array}{l}2 \text { opciones: } \\
1.03 \\
5 \text { opciones: } \\
0.23\end{array}$ & $\begin{array}{l}2 \text { opciones: TDAH } \\
\text { desempeño }<\text { que GC } \\
5 \text { opciones: } \text { TDAH= GC }\end{array}$ \\
\hline 20. & $\begin{array}{l}\text { CGT, versión } \\
\text { modificada }\end{array}$ & Suma de la apuesta & $\begin{array}{l}\text { Con FB: } 67.71 \\
(21.55) \\
\text { Sin FB: } 66.90 \\
(17.31)\end{array}$ & $\begin{array}{l}\text { Con FB: } 51.05 \\
(22.17) \\
\text { Sin FB:72.43 } \\
(14.01)\end{array}$ & $\begin{array}{l}\text { Con FB: } \\
0.76 \\
\text { Sin FB: } 0.35\end{array}$ & $\begin{array}{l}\text { TDAH desempeño }<\text { que GC } \\
\text { con FB }\end{array}$ \\
\hline
\end{tabular}

Nota: GC: grupo control. TDAH+AD: TDAH + ansiedad y depresión. FB: feedback. 
desempeño de los niños con TDAH y los controles en el primer juego, en el cual las primeras rondas se consideran como una fase de orientación, pero si en el segundo, en el cual se considera que todas las rondas evalúan TD bajo riesgo, presentando los niños con TDAH un comportamiento más arriesgado. Posteriormente, los mismos autores al analizar el comportamiento de niños con TDAH con la tarea Make a Match Game (Drechsler et al., 2010), encontraron que los niños con TDAH optan con mayor frecuencia por las recompensas menos probables, pero de mayor tamaño, sin diferir con los controles en la cantidad media de caramelos ganados. Adicionalmente, los autores informan que ninguno de los grupos cambió su estrategia durante la progresión de la tarea, y que las estrategias de TD no están relacionadas con la edad.

Kroyzer, Gross-Tsur, y Pollak (2014) informan que los adolescentes con TDAH realizan apuestas menores y eligen la opción menos probable con más frecuencia que el grupo control independientemente del subtipo, sexo, condiciones comórbidas, y el nivel intelectual, pero presentan la misma velocidad al tomar decisiones. El estudio de Pollak y Shoham (2015) examinó el comportamiento de adolescentes con TDAH en condiciones con y sin retroalimentación, la cual consistía en un mensaje que informaba el resultado de la apuesta (Ganó/Perdió), y observaron peor desempeño en condiciones con retroalimentación, evidenciando que la retroalimentación compromete, en lugar de mejorarla TD en el grupo con TDAH.

Por otro lado, entre los estudios con diferencias significativas que utilizaron pruebas de TD bajo incertidumbre, el de Toplak et al. (2005) informa que los adolescentes con TDAH hicieron elecciones menos ventajosas, pero no observaron diferencias entre los grupos en la puntuación final. Asimismo, no encontraron diferencias entre dos subtipos de TDAH (TDAH-C y TDAH-I), aunque los niños del grupo con TDAH-C parecen ser más sensibles a la frecuencia de las sanciones y menos sensibles a la magnitud de las mismas en comparación con los individuos del grupo TDAH-I. El estudio de Garon et al. (2006) obtuvo evidencias de que el subgrupo de niños con TDAH sin comorbilidad de síntomas de ansiedad y depresión muestra un rendimiento inferior al grupo con comorbilidad y al grupo control, sin demostrar aprendizaje durante el curso del juego.

Luman et al. (2008) indicaron que los niños con TDAH presentan un estilo de respuesta desadaptativa en comparación con los controles evidenciada por una preferencia menor por la alternativa ventajosa, cuando las penalizaciones aumentan en magnitud; mientras que cuando las penalizaciones aumentan en frecuencia, los niños con TDAH alcanzan un desempeño similar a los controles. Otro estudio (Hobson, Scott, y Rubia, 2011) que examinó el comportamiento de adolescentes con TDAH en la segunda parte del IGT, en la que los participantes tienen algún conocimiento del riesgo de sus opciones, encontró evidencias de que los niños con TDAH toman decisiones más riesgosas que los niños del grupo control. Humphreys y Lee (2011) encontraron que los niños con comorbilidad asumen mayor riesgo, seguido por los niños con TDAH sin comorbilidad y, por último, los niños del grupo control. En cuanto a la sensibilidad al castigo, los niños con comorbilidad parecen ser más sensibles al castigo, seguidos del grupo control, y luego los niños con TDAH sin comorbilidad. La edad se asoció significativamente con el total de pérdidas, pero el sexo no se asoció significativamente con ninguna de las variables de resultado.

Sallum, Mata, Miranda, y Malloy-Diniz (2013) encontraron que los niños y adolescentes con y sin TDAH presentan un desempeño similar en las puntuaciones típicas del test, sin embargo, existen diferencias en las estrategias que utilizan durante la prueba, respecto a mantenerse o cambiar la elección del mazo, ya que los niños y adolescentes con TDAH muestran un patrón de desplazamiento mayor del mazo ventajoso al desventajoso.

Patros et al. (2015) hallaron que los niños con TDAH respondieron más impulsivamente que los niños sin TDAH durante una tarea de dos opciones, pero no durante una tarea de cinco opciones, sustentando la idea de que la impulsividad relacionada con el TDAH no es omnipresente, sino que depende de la variación de la demanda y / o el contexto.

En la Tabla 4 se presentan los nueve (45\%) estudios que encontraron resultados semejantes, sin diferencias significativas, en ambas muestras en las pruebas de toma de decisiones.

Como puede observarse, los resultados del $45 \%$ de los estudios (9 de 20) seleccionados para este trabajo, no han encontrado evidencias de diferencias significativas en los desempeños de los niños y adolescentes con y $\sin$ TDAH.

Dos investigaciones analizaron la TD bajo riesgo (Scheres et al, 2006; DeVito, et al., 2008). El estudio de Scheres et al., refiere que podría haber una baja sensibilidad de la tarea asociada a varios factores, por un lado, la magnitud mayor de la recompensa en comparación con otras tareas similares y la expectativa de ganar una gran recompensa que podría aumentar la motivación de los adolescentes con TDAH y facilitar la espera, y por otro lado el hecho de brindar a los adolescentes una recompensa en la etapa de prueba que también podría interferir en la TD posterior. Y el estudio de De Vito et al. 
Tabla 4. Resultados de los estudios que obtuvieron puntajes similares en ambos grupos

\begin{tabular}{|c|c|c|c|c|c|c|}
\hline Estudio & Prueba de TD & Medida de salida & $\begin{array}{l}\text { M grupo control } \\
(D T)\end{array}$ & $\begin{array}{l}\text { M grupo clínico } \\
(D T)\end{array}$ & $d$ & $\begin{array}{l}\text { Resumen de } \\
\text { resultados }\end{array}$ \\
\hline 3. & HDT & $\begin{array}{c}\text { Cantidad de } \\
\text { elecciones de } \mathrm{c} / \text { puerta }\end{array}$ & $\mathrm{S} / \mathrm{D}$ & $\mathrm{S} / \mathrm{D}$ & $\mathrm{S} / \mathrm{D}$ & $\mathrm{TDAH}=\mathrm{GC}$ \\
\hline 4. & $\begin{array}{c}\text { Tareas de } \\
\text { descuento } \\
\text { temporal y } \\
\text { probabilístico }\end{array}$ & $\begin{array}{c}\text { Demora en segundos } \\
\text { y niveles de } \\
\text { probabilidad }\end{array}$ & $\mathrm{S} / \mathrm{D}$ & $\mathrm{S} / \mathrm{D}$ & $\mathrm{S} / \mathrm{D}$ & $\mathrm{TDAH}=\mathrm{GC}$ \\
\hline 5. & CGT & $\begin{array}{c}\text { Elecciones racionales, } \\
\text { cantidad de apuesta, } \\
\text { índice de riesgo de } \\
\text { ajuste }\end{array}$ & $\mathrm{S} / \mathrm{D}$ & $\mathrm{S} / \mathrm{D}$ & $\mathrm{S} / \mathrm{D}$ & $\begin{array}{l}\text { TDAH=GC; } \\
\text { TDAH desempeño } \\
<\text { que TDAH-Me- } \\
\text { tilfenidato }\end{array}$ \\
\hline 8. & IGT niños & $\begin{array}{c}\mathrm{N}^{\circ} \text { total de elecciones } \\
\text { ventajosas }\end{array}$ & $\mathrm{S} / \mathrm{D}$ & $\mathrm{S} / \mathrm{D}$ & $\mathrm{S} / \mathrm{D}$ & $\mathrm{TDAH}=\mathrm{GC}$ \\
\hline 10. & HDT & $\begin{array}{c}\text { Fórmula = puertas } \\
\text { ventajosas - puertas } \\
\text { desventajosas a lo } \\
\text { largo de los } 10 \text { bloques }\end{array}$ & $\mathrm{S} / \mathrm{D}$ & $\mathrm{S} / \mathrm{D}$ & $\mathrm{S} / \mathrm{D}$ & $\begin{array}{l}\text { TDAH }(\text { con y sin } \\
\text { déficit de } F E)=G C\end{array}$ \\
\hline 14. & HDT & $\begin{array}{l}\text { Elecciones seguras y } \\
\text { elecciones de riesgo }\end{array}$ & 5.08 (S/D) & $\begin{array}{l}\text { TDAH-C: } 4.91 \\
\text { TDAH-I: } 4.86 \\
\text { (S/D) }\end{array}$ & $\mathrm{S} / \mathrm{D}$ & $\mathrm{TDAH}=\mathrm{GC}$ \\
\hline 16. & HDT & $\begin{array}{l}\text { Elecciones seguras y } \\
\text { elecciones de riesgo }\end{array}$ & $4.9(33.1)$ & $4.7(37)$ & -0.00 & $\mathrm{TDAH}=\mathrm{GC}$ \\
\hline 17. & IGT niños & $(\mathrm{C}+\mathrm{D})-(\mathrm{A}+\mathrm{B})$ & $-7.07(15.43)$ & $\begin{array}{l}\text { TDAH: } 0.97 \\
\quad(19.52) \\
\text { TDAH +TND: } \\
-2.39(22.31)\end{array}$ & $\begin{array}{c}\text { TDAH: }-0.46 \\
\text { TDAH+TND: } 0.49\end{array}$ & $\begin{array}{c}\mathrm{TDAH}=\mathrm{TDAH}+\mathrm{T}- \\
\mathrm{ND}=\mathrm{GC}\end{array}$ \\
\hline 18. & HDT & $\begin{array}{l}\text { Elecciones seguras y } \\
\text { elecciones de riesgo }\end{array}$ & $\begin{array}{l}\text { Seguras: } 14(9.5) \\
\text { Riesgo: } 18(8.6)\end{array}$ & $\begin{array}{l}\text { Seguras: } 13(7.8) \\
\text { Riesgo: } 20(10.8)\end{array}$ & $\begin{array}{l}\text { Seguras: } 0.11 \\
\text { Riesgo: }-0.21\end{array}$ & $\mathrm{TDAH}=\mathrm{GC}$ \\
\hline
\end{tabular}

Nota: S/D: sin dato; GC: grupo control; TDAH+AD: TDAH + ansiedad y depresión.

(2008) evaluó los efectos de la medicación estimulante sobre la TD en el TDAH, encontrando que la apuesta del grupo con TDAH fue más conservadora en la sesión con la medicación que en la sesión de placebo, pero sin diferencias en el número de sus elecciones racionales o el ajuste de riesgo.

Y siete $(50 \%)$ de los 14 estudios que analizaron la TD bajo incertidumbre (Antonini, Becker, Tamm, y Epstein, 2015; Geurts, Van der Oord, \& Crone, 2006; Hovik et al., 2015; Lambek et al., 2011; Masunami, Okazaki y Maekawa, 2009; Skogli, Egeland, Andersen, Hovik y Oie 2013; Skogli, Andersen, Hovik, y Oie 2014) no encontraron evidencias de diferencias en el desempeño de los niños y adolescentes con TDAH y los niños del grupo control.

Es de destacar que ninguno de los 5 trabajos que utilizó la prueba HDT encontró diferencias entre los grupos. Este conjunto representa el $25 \%$ de los estudios in- cluidos en este análisis y el 55\% de las investigaciones que no encontraron diferencias en el desempeño de niños y adolescentes con y sin TDAH.

El estudio de Masunami et al. (2009) no encontró diferencias en el número de elecciones ventajosas, aunque si encontraron diferencias en los patrones de tiempo (T-patterns) que están relacionados con la sensibilidad a las recompensas y penalidades. Los resultados mostraron que hubo significativamente menos patrones de tiempo, incluidas las sanciones, en los niños con TDAH en comparación con los controles, lo que indicaría que los niños con TDAH son menos sensibles a las sanciones, prestando menos atención a las mismas que los del grupo control.

Skogli et al. (2013) realizaron el estudio en niños y adolescentes con TDAH de subtipo combinado (TDAH-C), subtipo inatento (TDAH-I), y un grupo control, y si bien no encontraron diferencias significativas, 
observaron elecciones más desventajosas en los niños menores de 12 años y un mejor rendimiento de TD a medida que aumentaba la edad, lo que podría explicarse como un efecto del desarrollo.

\section{Discusión}

La presente revisión evidencia la heterogeneidad de las investigaciones sobre la TD en niños y adolescentes con TDAH, particularmente en el tipo de toma de decisiones, en el tipo de tareas utilizadas, en la composición de las muestras y, especialmente, en los resultados, que no son concluyentes y mantienen la controversia sobre el tema. Esto mismo se ha constatado en otras revisiones con niños y con población adulta (Dekkers, Popma, Van Rentergem, Bexkens, y Huizenga, 2016; Groen et al., 2013).

El objetivo de esta revisión fue analizar sistemáticamente resultados de investigaciones sobre el desempeño de niños y adolescentes con diagnóstico de TDAH en tareas de toma de decisiones. El 55\% de los 20 estudios incluidos en este análisis aportó evidencias de que los individuos con TDAH muestran un comportamiento más arriesgado y/o toman decisiones desventajosas en tareas de juego, por consiguiente, sostienen que los niños y adolescentes con TDAH parecerían más propensos a arriesgarse o a tomar decisiones desfavorables. Sin embargo, el $45 \%$ restante de los estudios analizados, no encontró evidencias suficientes para sustentar estas afirmaciones, lo que, a nuestro criterio, mantiene la incertidumbre sobre los efectos e influencias del TDAH en los procesos de TD bajo riesgo o incertidumbre. Dada esta diferencia en los resultados analizamos la posibilidad de que la alta variabilidad observada en factores como el tamaño y la composición de la muestra los instrumentos y las tareas utilizadas, la presencia de comorbilidad, el uso de medicación, las diferencias de CI, entre otros, permitieran explicar estas inconsistencias.

Con relación a las características de los participantes, específicamente con la edad, dos tercios de los estudios (6 de 9) que han evaluado únicamente a niños con edades entre 6 y 13 años, informaron desempeño inferior en los niños del grupo clínico (Drechsler et al., 2008; Drechsler et al., 2010; Garon et al., 2006; Luman et al., 2008; Humphreys y Lee, 2011; Patros et al., 2015). En sentido contrario, el 75\% (6 de 8 ) de los estudios que trabajaron con muestras mixtas de niños y de adolescentes no encontraron diferencias en las medias de los desempeños de ambos grupos (Hovik et al., 2015; Lambek et al., 2011; Masunami et al., 2009; Scheres et al., 2006; Skogli et al., 2014; Skogli et al., 2013). Y la totalidad de los estudios (3) que analizaron sólo el desempeño en adolescentes (Kroyzer et al., 2014; Pollak y Shoham, 2015; Toplak et al., 2005), encontraron evidencias de un desempeño inferior de los jóvenes con TDAH. Los resultados respecto a lo que sucede con los adolescentes son consistentes con los estudios de Bernal, Montaña, Acosta y Rojas (2015), que describen que a partir de los 14 años, a pesar de los avances en el funcionamiento cognitivo, no se observa una mejora gradual en el desempeño en la toma de decisiones, posiblemente debido a un aumento de la búsqueda de sensaciones, a la adopción de comportamientos de riesgo y a la tendencia a alejarse de la norma, afectando el proceso de TD (Reyna y Farley, 2006).

Respecto al género, la totalidad de los estudios evaluaron mayormente niños y adolescentes de género masculino, ya que la prevalencia del trastorno es mayor en niños que en niñas (APA, 2013), sin embargo, no se observaron diferencias en cuanto al desempeño en la toma de decisiones.

Con relación al $C I$, algunos estudios no expresan que haya sido considerado (estudios 5, 8, 12 y 13). La mayoría de los estudios que lo controlaron, conformaron muestras con niños y adolescentes con TDAH que presentan un CI más bajo que los pares del grupo control, con diferencias significativas en 6 de ellos (estudio 7, 10, $11,16,17$ y 19) y con peor desempeño de los niños del grupo clínico, lo cual puede afectar los resultados del desempeño en toma de decisiones, ya que los resultados podrían explicarse por las diferencias iniciales en el CI.

Otras de las variables a considerar es la presencia de comorbilidad, uno de los estudios presenta evidencias de que los niños con TDAH con trastorno de ansiedad-depresión son más sensibles a los refuerzos, en particular a la pérdida, por lo cual su desempeño en tareas de toma de decisión se vuelve más conservadora, lo que implicaría que la presencia de un trastorno de este tipo podría tener un efecto protector en los niños con TDAH (Garon et al., 2006). Otro estudio encontró que el grupo TDAH con comorbilidad con un trastorno negativista desafiante (TND) asume más riesgos y parece ser más sensible al castigo (Humphreys y Lee, 2011). Los demás estudios que consideraron las comorbilidades, no encontraron diferencias en los niños TDAH con y sin comorbilidad (Antonini et al., 2015; Kroyzer et al., 2014; Lambek et al., 2011). Además, es importante destacar que los estudios que encontraron diferencias significativas en tareas de toma de decisiones, no controlaron la variable comorbilidad, lo cual, considerando los efectos de la presencia de comorbilidades con algunos trastornos, podría conducir a resultados o conclusiones erróneas.

Por último, con relación a la presencia de diagnóstico de subtipo de TDAH, dos estudios lo incluyeron en sus 
análisis, Toplak et al. (2005) informaron que no hay diferencias entre los subtipos, y Skogli et al. (2013) si bien no encontraron diferencias entre los subtipos en general, sugieren la posibilidad de que las diferencias entre los grupos pueden ser más evidentes con el aumento de las edades, con una mayor cantidad de elecciones desventajosas en el subtipo TDAH-C.

Al considerar las características metodológicas de las investigaciones incluidas en esta revisión, por ejemplo, el tamaño de los grupos estudiados, encontramos que el $50 \%$ de los estudios trabajó con muestras menores a $\mathrm{N}=$ 30 , lo que aumenta la probabilidad de que algún sesgo influya en los resultados.

En lo que se refiere al tipo de tarea de utilizada, los estudios se dividen entre la aplicación de tareas bajo incertidumbre $(70 \%)$ y bajo riesgo (30\%). El $45 \%$ de los estudios que utilizaron pruebas de TD bajo incertidumbre encontraron evidencias de TD desventajosas en los niños y adolescentes con TDAH, y el 55\% restante mostró desempeños similares en los participantes con y sin TDAH. Las pruebas de TD bajo incertidumbre se asocia a una dificultad en el procesamiento de la recompensa y el castigo relacionado básicamente con el control inhibitorio (Seguin, Arseneault, y Tremblay, 2007). Es interesante resaltar que el $71 \%$ de los estudios que no encontraron diferencias en el desempeño de niños y adolescentes con y sin TDAH en las tareas de TD bajo incertidumbre, utilizaron para la evaluación el HDT. Coincidimos con lo propuesto por Skogli et al. (2013) que afirman que en el HDT el niño puede sentirse menos implicado en la tarea y menos motivado al decidir por un «burro» que es quien recibe las recompensas y no por sí mismos. Es necesario alertar sobre la escasa sensibilidad de este instrumento para discriminar diferencias en tareas de TD bajo incertidumbre.

Por otro lado, el $67 \%$ de los estudios que utilizaron pruebas de TD bajo riesgo encontraron evidencias de TD desventajosas en niños y adolescentes con TDAH. Estas pruebas se vinculan estrechamente con capacidades como la comprensión de las probabilidades, la posibilidad de actualizar estos conocimientos en la memoria de trabajo y almacenarla en la memoria a largo plazo, y ser capaces de inhibir las respuestas a los comentarios (Van Duijvenvoorde, Bredman, y Huizenga, 2012).

Con relación a la cantidad de ensayos, se encontró que es muy variable entre las diferentes pruebas y estudios $\mathrm{y}$, en general, no consideran el factor fatiga ni la distracción en una población con déficit en la atención. Los niños con TDAH tienden a realizar mayor cantidad de elecciones cuyas consecuencias son inmediatas (Antrop et al., 2006) y la preferencia por estas consecuencias se asocian con la falta de atención (Paloyelis, Asher- son, y Kuntsi, 2009). Bajo ciertas condiciones, los niños con este trastorno parecen ser más sensibles a la frecuencia de castigo que a su magnitud (Luman et al., 2008) y prestan más atención a las recompensas que al castigo (Masunami et al., 2009). Por lo tanto, resultaría aconsejable manejar o estabilizar el número de ensayos utilizados en las tareas, a los fines de poder controlar los efectos de la fatiga y la distractibilidad.

Otras de las variables que podrían ser fuente de controversia es la cantidad de opciones para elegir en las pruebas, en las que también se observa una gran variabilidad, con mayoría de pruebas que proponen cuatro opciones para elegir. Las tareas con más de dos opciones son más similares a lo que sucede en la vida real, ya que los niños, fuera del entorno de la evaluación, suelen tener una gama de opciones de elección, y no dos únicamente, por lo tanto, una tarea experimental que presenta una medida continua de TD podría reflejar mejor el proceso y sus resultados (Patros et al., 2015).

Respecto al tipo de recompensas, el análisis de los estudios nos ha permitido establecer que aquellas que no fueron reales tienden a generar falta de motivación externa que pueda atenuar la propensión a las decisiones más arriesgadas de los niños con TDAH (Garon et al., 2006). Si bien no hay estudios sobre TDAH que analicen la diferencia entre ambos tipos de recompensas (reales y ficticias), es importante destacar que de los estudios que no encontraron diferencias entre niños y adolescentes con y sin TDAH, solo uno de ellos contó con recompensas reales, y el resto fueron realizados con manzanas ficticias y dinero ficticio.

Algunos estudios utilizaron la prueba de conciencia, la cual consiste en realizar preguntas a los participantes, luego de la administración de la tarea, para evaluar el reconocimiento y la comprensión de las opciones ventajosas y desventajosas. Los tres estudios que mencionan el haber administrado la prueba de conciencia fueron el de Garon et al. (2006), en el cual el grupo con TDAH mostró menor conciencia del juego que el grupo control y el grupo con TDAH con síntomas de ansiedad y depresión; el estudio de Toplak et al. (2005), que informa que los participantes de ambos grupos generalmente comprendían la tareas y reconocían las opciones ventajosas y desventajosas; y el estudio de Lambek et al. (2011), que indica que sólo tres niños no tuvieron noción de lo que estaba ocurriendo en el HDT, mientras que el 38\% de los niños presentaron una preferencia por la estrategia equivocada (puertas desventajosas), y el 58\% un presentimiento o una formulación correcta de los conocimientos acerca de por qué las puertas A y B eran desventajosas y las puertas C y D ventajosas en el largo plazo. Los demás estudios no administraron la prueba de conciencia, 
o bien, no lo informan en la publicación. Estos resultados son inconsistentes respecto a la conciencia, por lo que sería interesante profundizar en este aspecto en futuros estudios, con el objeto de determinar de forma sistemática si los niños y adolescentes con TDAH realmente reconocen las opciones ventajosas y desventajosas y toman decisiones considerando las posibles consecuencias de sus elecciones, o si tan sólo emiten de forma precipitada sus respuestas, automáticas, y determinadas por la impulsividad característica del trastorno.

A pesar de que podría existir relación entre el riesgo real, el rendimiento en tareas de juego y el TDAH en la infancia, no se dispone de conocimientos concluyentes acerca de la relación entre el desempeño en estas tareas y el comportamiento en la vida cotidiana. Algunos de los estudios revisados sugieren que existe una asociación entre el rendimiento en tareas de apuestas arriesgadas y la severidad de los síntomas del TDAH (Drechsler et al., 2008; Hobson et al., 2011; Toplak et al., 2005). Sin embargo, no hay estudios sobre la validez ecológica de la ejecución de tareas de juego en los niños y adolescentes, por lo cual sería necesario investigarlo.

Dos estudios incluyeron el tratamiento farmacológico a los participantes con TDAH (De Vito, 2008; Hovik, 2015) e investigaron los efectos del mismo en la ejecución de tareas de toma de decisiones, descubriendo que genera un juego más conservador; sin embargo, dado el reducido número de estudios, no se pueden obtener conclusiones sólidas sobre la eficacia del fármaco en la reducción de comportamientos de riesgo en tareas de toma de decisiones.

Además, son escasos los estudios ecológicos sobre la TD (Katsikopoulos y Lan, 2011), con poco avance del conocimiento acerca de la relación entre el rendimiento en este tipo de tareas y el comportamiento en la vida real, siendo pocos los estudios que sugieren que el desempeño de los niños con TDAH en tareas de TD se vincula con la severidad de los síntomas (Drechsler, et al., 2008; Hobson, et al., 2011; Toplak et al., 2005).

En la presente revisión se identificaron algunos aspectos que restringen la generalización de los resultados como, por ejemplo, falta de unificación de los criterios de clasificación de los componentes de la función ejecutiva, el empleo de diseños de estudio que no permiten demostrar causalidad y el uso de muestreos no probabilísticos. Estos aspectos, que Boyle, Connolly, y MacKay (2016) consideran característicos de los artículos que denominaron «literatura gris», dificultan la realización de revisiones metaanalíticas sobre la TD en TDAH, sin embargo, se considera que un futuro metaanálisis podría aportar evidencia complementaria a este estudio sistemático del estado de la cuestión.
Entre las limitaciones del presente estudio es importante mencionar que no se han incluido aquellos estudios no publicados en revistas científicas o tesis de grado y postgrado que trabajan en la temática, y que no se han podido realizar análisis estadísticos de comparación entre los estudios, debido a que no todos publicaron la totalidad de sus resultados.

Esta revisión constituye un intento sistematizado para reunir la evidencia que refleje el estado del conocimiento y las lagunas en el estudio de la TD en niños y adolescentes con TDAH, fenómeno que afecta seriamente la salud de este grupo poblacional y que implica un alto costo social y económico, por lo que representa una prioridad de acción para los profesionales de la salud. La evidencia presentada, si bien sugiere que la TD es un proceso que se encuentra alterado en el TDAH, resulta un claro indicador de que aún no se cuenta con datos concluyentes al respecto. También se podría sostener la idea de que no se trate de una alteración o de un déficit en el proceso, sino que se podría hablar de estilos diferentes de TD, ampliando la visión desde una perspectiva positiva. Lógicamente, esto supondría implicaciones y cambios para la práctica clínica y el rol del tratamiento neuropsicológico.

Al trabajar con tareas de TD en niños y adolescentes con y sin TDAH, no debería perderse de vista que los estudios no han encontrado evidencias suficientes que permitan sustentar la idea de diferencias (55\% vs $45 \%$ ) y destacar la importancia de controlar el CI, la presencia o ausencia de comorbilidad, el uso de medicación y los subtipos de TDAH.

Finalmente, las evidencias permiten sugerir que los estudios sobre el desempeño de niños y adolescentes con y $\sin$ TDAH tendrían mayor validez y confiabilidad si trabajaran con muestras mayores a 30 sujetos; si incluyeran y compararan desempeños en tareas de TD tanto bajo riesgo como bajo incertidumbre; si consideraran la distractibilidad y la fatiga de los niños con TDAH al calcular la cantidad de ensayos; si utilizaran tareas ecológicas, diseñadas con más de dos opciones de respuesta y recompensas reales; y si se incluyera de forma sistemática la prueba de conciencia para diferenciar las respuestas automáticas e impulsivas de las que implican un análisis controlado.

\section{Referencias}

* Las referencias marcadas con asterisco indican los estudios incluidos en la revisión sistemática.

American Psychiatric Association (APA). (2013). DSM-5. Manual diagnóstico y estadístico de los trastornos mentales. Barcelona: Masson. 
*Antonini, T. N., Becker, S. P., Tamm, L., \& Epstein, J. N. (2015). Hot and cool executive functions in children with attention-deficit/hyperactivity disorder and comorbid oppositional defiant disorder. Journal of the International Neuropsychological Society, 21, 584-595. doi:10.1017/S1355617715000752.

Antrop, I., Stock, P., Verté, S., Wiersema, J. R., Baeyens, D., \& Roeyers, H. (2006). ADHD and delay aversion: the influence of non-temporal stimulation on choice for delayed rewards. Journal of Child Psychology and Psychiatry, 47, 11521158. doi: 10.1111/j.1469-7610.2006.01619.x.

Barkley, R. A. (2010). Differential diagnosis of adults with ADHD: the role of executive function and self-regulation. Journal of Clinical Psychiatry, 71, 17. doi: 10.4088/JCP.9066tx1c.

Barkley, R. A. (2011). Is Executive Functioning Deficient in ADHD? It depends on your definitions and your measures. The ADHD Report, 19, 1-10.

Barkley, R. A. (2013). Taking charge of ADHD: The complete, authoritative guide for parents. New York: Guilford Publications.

Bechara A., Damasio A. R, Damasio H., \& Anderson S. W. (1994). Insensitivity to future consequences following damage to human prefrontal cortex. Cognition, 50, 7-15. doi: 10.1016/00100277(94)90018-3.

Bechara, A. (2004). The role of emotion in decision-making: Evidence from neurological patients with orbitofrontal damage. Brainand Cognition, 55,30-40.doi:10.1016/j.bandc.2003.04.001.

Bechara, A., Damasio, H., \& Damasio, A. R. (2000). Emotion, decision making and the orbitofrontal cortex. Cerebral Cortex, 10, 295-307. doi: 10.1093/cercor/10.3.295.

Bernal, P., Montaña, J., Acosta, R., \& Rojas, Y. (2015). Performance of Children and Adolescents from a School of the City of Sogamoso on a Decision-Making Test. Open Journal of Pediatrics, 5, 339-347. doi: 10.4236/ojped.2015.54051.

Boyle, J., Connolly, M., \& MacKay, T. (2016). Systematic review and meta-analysis. Educational \& Child Psychology, 33, 7691.

Brand, M., Fujiwara, E., Borsutzky, S., Kalbe, E., Kessler, J., \& Markowitsch, H. J. (2005) Decision making deficits of korsakoff patients in a new gambling task with explicit rules: Associations with executive functions. Neuropsychology, 19, 267277. doi: 10.1037/0894-4105.19.3.267.

Brand, M., Grabenhorst, F., Starcke, K., Vandekerckhove, M. M. P., \& Markowitsch, H. J. (2007). Role of the amygdala in decisions under ambiguity and decisions under risk: Evidence from patients with Urbach-Wiethe disease. Neuropsychologia, 45, 1305-1317. doi: 10.1016/j.neuropsychologia.2006.09.021.

Brand, M., Heinze, K., Labudda, K., \& Markowitsch, H. J. (2008). The role of strategies in deciding advantageously in ambiguous and risky situations. Cognitive Processing, 9, 159-173. doi: 10.1007/s10339-008-0204-4.

Camprodon, E., Duñó, L., Batlle, S., Estrada, X., Acena, M., Marrón, M., ... Torrubia, R. (2013). El tempo cognitivo lento: Revisión de un constructo. Revista de Psicopatología y Psicología Clínica, 18, 151-168. doi: 10.5944/rppc.vol.18. num.2.2013.12771.

Cardo, E., y Amengual-Gual, M. (2015). ¿Se asocia el trastorno por déficit de atención/hiperactividad con otras patologías prevalentes de la infancia?. Revista de Neurología, 60, 109113.
Cardo, E., y Servera, M. (2008). Trastorno por déficit de atención/ hiperactividad: estado de la cuestión y futuras líneas de investigación. Revista de Neurología, 46, 365-372.

Castellanos, F. X., \& Tannock, R. (2002). Neuroscience of attention-deficit/hyperactivity disorder: the search for endophenotypes. Nature Reviews Neuroscience, 3, 617-628. doi:10.1038/ nrn896.

Catelan-Mainardes, S. C. (2010). Transtorno de déficit de atenção e hiperatividade na infância e adolescência pela perspectiva da neurobiologia. Revista Saúde e Pesquisa, 3, 385-391.

Cohen, J. (1988). Statistical Power Analysis for the Behavioral Sciences (2nd Ed.). Hillsdate, NJ, LEA.

Crone, E. A., \& Van der Molen, M. W. (2004). Developmental changes in real life decision making: performance on a gambling task previously shown to depend on the ventromedial prefrontal cortex. Developmental Neuropsychology, 25, 251279. doi: 10.1207/s15326942dn2503_2.

Damasio, A. R., Everitt, B. J., \& Bishop, D. (1996). The somatic marker hypothesis and the possible functions of the prefrontal cortex [and discussion]. Philosophical Transactions of the Royal Society of London B: Biological Sciences, 351, 14131420 .

Dekkers, T. J., Popma, A., Van Rentergem, J. A. A., Bexkens, A., \& Huizenga, H. M. (2016). Risky decision making in Attention-Deficit/Hyperactivity Disorder: A meta-regression analysis. Clinical Psychology Review, 45, 1-16. doi: 10.1016/j. cpr.2016.03.001.

*DeVito, E. E., Blackwell, A. D., Kent, L., Ersche, K. D., Clark, L., Salmond, C. H., ... Sahakian, B. J. (2008). The effects of methylphenidate on decision making in attention-deficit/hyperactivity disorder. Biological Psychiatry, 64, 636-639. doi: 10.1016/j.biopsych.2008.04.017.

*Drechsler, R., Rizzo, P., \& Steinhausen, H. C. (2008). Decision-making on an explicit risk-taking task in preadolescents with attention-deficit/hyperactivity disorder. Journal of Neural Transmission, 115, 201-209. doi: 10.1007/s00702-0070814-5.

*Drechsler, R., Rizzo, P., \& Steinhausen, H. C. (2010). Decision making with uncertain reinforcement in children with attention deficit/hyperactivity disorder (ADHD). Child Neuropsychology, 16, 145-161. doi: 10.1080/09297040903190774.

García-Berjillos, E., Aliño, M., Gadea, M., Espert, R., \& Salvador, A. (2015). Eficacia del neurofeedback para el tratamiento de los trastornos del espectro autista: Una revisión sistemática. Revista de Psicopatología y Psicología Clínica, 20, 151163.

*Garon, N., Moore, C., \& Waschbusch, D. A. (2006). Decision making in children with ADHD only, ADHD-anxious/depressed, and control children using a child version of the Iowa Gambling Task. Journal of Attention Disorders, 9, 607-619. doi: 10.1177/1087054705284501.

*Geurts, H. M., Van der Oord, S., \& Crone, E. A. (2006). Hot and cool aspects of cognitive control in children with ADHD: Decision-making and inhibition. Journal of abnormal child psychology, 34, 811-822. doi: 10.1007/s10802-006-9059-2.

Groen, Y., Gaastra, G. F, Lewis-Evans, B., \& Tucha, O. (2013). Risky behavior in Gambling Tasks in individuals with ADHD - A systematic literature review. PLOS ONE, 8, 74909. doi: 10.1371/journal.pone.0074909. 
*Hobson, C. W., Scott, S., \& Rubia, K. (2011). Investigation of cool and hot executive function in ODD/CD independently of ADHD. Journal of Child Psychology and Psychiatry, 52, 1035-1043. doi: 10.1111/j.1469-7610.2011.02454.x.

*Hovik, K. T., Plessen, K. J., Cavanna, A. E., Skogli, E. W., Andersen, P. N., \& Oie, M. (2015). Cognition, Emotion and Behavior in Children with Tourette's Syndrome and Children with ADHD-Combined Subtype-A Two-Year Follow-Up Study. 10, 1-22.

*Humphreys, K. L., \& Lee, S. S. (2011). Risk taking and sensitivity to punishment in children with ADHD, ODD, ADHD+ODD, and controls. Journal of Psychopathology and Behavioral Assessment, 33, 299-307. doi: 10.1007/ s10862-011-9237-6.

Kahneman, D. (2011). Thinking, fast and slow. New York: Farrar, Straus, \& Giroux.

Kahneman, D., \& Tversky, A. (1984). Choices, values, and frames. American Psychologist, 39, 341-350.

Katsikopoulos, K. V. \& Lan, C. H. (2011). Herbert Simon's spell on judgment and decision making. Judgment and decision making, 6, 722 .

*Kroyzer, N., Gross-Tsur, V., \& Pollak, Y. (2014). Risk taking in adolescents with attention deficit hyperactivity disorder on a probabilistic choice task. The Journal of nervous and mental disease, 202,247-252.doi:10.1097/NMD.0000000000000100.

*Lambek, R., Tannock, R., Dalsgaard, S., Trillingsgaard, A., Damm, D., \& Thomsen, P. H. J. (2011). Executive dysfunction in school-age children with ADHD. Journal of Attention Disorder, 15, 646-655. doi: 10.1177/1087054710370935.

Lee, S. S., Humphreys, K. L., Flory, K., Liu, R., \& Glass, K. (2011). Prospective association of childhood attention-deficit/ hyperactivity disorder (ADHD) and substance use and abuse/ dependence: a meta-analytic review. Clinical Psychology Review, 31, 328-341. doi: 10.1016/j.cpr.2011.01.006.

Lejuez C. W., Read J. P., Kahler C. W., Richards J. B., Ramsey S. E., Stuart, G. L., ... Brown, R. A. (2002) Evaluation of a behavioral measure of risk taking: The balloon analogue risk task (BART). Journal of Experimental Psychology: Applied, 8, 75-84. doi: 10.1037/1076-898X.8.2.75.

López-Martín, S., Albert, J., Fernández-Jaén, A., y Carretié, L. (2010). Neurociencia afectiva del TDAH: Datos existentes y direcciones futuras. Escritos de Psicología, 3, 17-29.

*Luman, M., Oosterlaan, J., Knol, D. L., \& Sergeant, J. A. (2008). Decision-making in ADHD: Sensitive to frequency but blind to the magnitude of penalty? Journal of Child Psychology and Psychiatry, 49, 712-722. doi: 10.1111/j.1469-7610.2008.01910.x.

Mannuzza, S., Klein, R. G., \& Moulton, J. L. (2008). Lifetime criminality among boys with attention deficit hyperactivity disorder: a prospective follow-up study into adulthood using official arrest records. Psychiatry Research, 160, 237-246. doi: 10.1016/j.psychres.2007.11.003.

*Masunami, T., Okazaki, S., \& Maekawa, H. (2009). Decision-making patterns and sensitivity to reward and punishment in children with attention-deficit hyperactivity disorder. International journal of psychophysiology, 72, 283-288. doi: 10.1016/j.ijpsycho.2009.01.007.

Mediavilla-García, C. (2003). Neurobiología del trastorno de hiperactividad. Revista de Neurología, 36, 555-565.
Mowinckel, A. M., Pedersen, M. L., Eilertsen, E., \& Biele, G. (2015). A meta-analysis of decision-making and attention in adults with ADHD. Journal of Attention Disorders, 19, 355-367.

Navarro, M. I., y García-Villamisar, D. A. (2011). Funcionamiento ejecutivo en el trastorno de déficit de atención con hiperactividad: una perspectiva ecológica de los perfiles diferenciales entre los tipos combinado e inatento. Revista de Psicopatología y Psicología Clínica, 16, 113-124. doi: 10.5944/rppc. vol.16.num.2.2011.1035D

Organización Mundial de la Salud (OMS). (1992). Clasificación Internacional de Enfermedades, Décima revisión (CIE-10). Ginebra.

Orwin, R. G. (1994). Evaluating coding decisions. En H. Cooper y L. V. Hedges (Eds.), The handbook of research synthesis (pp. 139-162). Nueva York, NY: Russell Sage Publications.

Paloyelis, Y., Asherson, P., \& Kuntsi, J. (2009). Are ADHD symptoms associated with delay aversion or choice impulsivity? A general population study. Journal of American Academy of Child and Adolescent Psychiatry, 48, 837-846. doi: 10.1097/ CHI.0b013e3181ab8c97.

*Patros, C. H., Alderson, R. M., Lea, S. E., Tarle, S. J., Kasper, L. J., \& Hudec, K. L. (2015). Visuospatial working memory underlies choice-impulsivity in boys with attention-deficit/hyperactivity disorder. Research in Developmental Disabilities, 38, 134-144. doi: 10.1016/j.ridd.2014.12.016.

Perestelo-Pérez, L. (2013). Standards on how to develop and report systematic reviews in Psychology and Health. International Journal of Clinical and Health Psychology, 13, 49-57. doi: 10.1080/09297049.2015.1119261.

*Pollak, Y., \& Shoham, R. (2015). Feedback may harm: Role of feedback in probabilistic decision making of adolescents with ADHD. Journal of Abnormal Child Psychology, 43, 12331242. doi: 10.1007/s10802-015-0016-9.

Quezada, C. (2007). Potencia estadística, sensibilidad y Tamaño de efecto: ¿un nuevo canon para la investigación? Onomázein, 16, 159-170.

Ramos-Loyo, J., Taracena, A, M., Sánchez-Loyo, L. M., Matute, E., y González-Garrido, A. A. (2011). Relación entre el funcionamiento ejecutivo en pruebas neuropsicológicas y en el contexto social en niños con TDAH. Revista Neuropsicología, Neuropsiquiatría y Neurociencias, 11, 1-16.

Reyna, V., \& Farley, F. (2006) Risk and Rationality in Adolescent Decisión Making: Implications for Theory Practice, and Public Policy. Psychological Science in the Public Interest, 7, 1-44. doi: 10.1111/j.1529-1006.2006.00026.x.

Rogers, R. D., Everitt, B. J., Baldacchino, A., Blackshaw, A. J., Swainson, R., Wynne, K., ... Robbins, T. W. (1999). Dissociable deficits in the decision-making cognition of chronic amphetamine abusers, opiate abusers, patients with focal damage to prefrontal cortex, and tryptophan-depleted normal volunteers: evidence for monoaminergic mechanisms. Neuropsychopharmacology, 20, 322-339. doi:10.1016/S0893133X(98)00091-8.

Rubiales, J., Bakker, L., y Delgado-Mejía, I. D. (2010). Organización y planificación en niños con TDAH: evaluación y propuesta de un programa de estimulación. Cuadernos de Neuropsicología, 5, 145-161.

Rubiales, J., Bakker, L., y Urquijo, S. (2013). Estudio comparativo del control inhibitorio y la flexibilidad cognitiva en niños 
con Trastorno por déficit de atención con hiperactividad. Revista Cuadernos de Neuropsicología, 7, 50-69.

*Sallum, I., Mata, F., Miranda, D. M., \& Malloy-Diniz, L. F. (2013). Staying and shifting patterns across IGT trials distinguish children with externalizing disorders from controls. Frontiers in psychology, 4, 899. doi: 10.3389/ fpsyg.2013.00899.

Sánchez-Encalada, L.,y Díaz, M. D. (2009). Detección de intervención a través del juego del TDAH. Revista de Psicología Iztacala 12, 156-170.

Sánchez-Meca, J., y Botella-Ausina, J., (2010). Revisiones sistemáticas y meta-análisis: Herramientas para la práctica profesional. Papeles del Psicólogo, 31, 7-17.

*Scheres, A., Dijkstra, M., Ainslie, E., Balkan, J., Reynolds, B., Sonuga-Barke, E., \& Castellanos, F. X. (2006). Temporal and probabilistic discounting of rewards in children and adolescents: Effects of age and ADHD symptoms. Neuropsychologia, 44, 2092-2103. doi: 10.1016/j.neuropsychologia.2005.10.012.

Seguin, J. R., Arseneault, L., \& Tremblay, R. E. (2007). The contribution of "cool" and "hot" components of decision-making in adolescence: Implications for developmental psychopathology. Cognitive Development, 22, 530-543. doi: 10.1016/j. cogdev.2007.08.006.

*Skogli, E. W., Andersen, P. N., Hovik, K. T., \& Oie, M. (2014). Development of Hot and Cold Executive Function in Boys and Girls With ADHD A 2-Year Longitudinal Study. Journal of Attention Disorders, 1-11. doi: 10.1177/1087054714524984.

*Skogli, E. W., Egeland, J., Andersen, P. N., Hovik, K. T., \& Oie, M. (2013). Few differences in hot and cold executive functions in children and adolescents with combined and inattentive subtypes of ADHD. Child Neuropsychology: A Journal on Normal and Abnormal Development in Childhood and Adolescence, 20, 1-20. doi: 10.1080/09297049.2012.753998.

Téllez-Villagra, C., Valencia-Flores, M., y Beauroyre-Hijar, R. (2011). Cronología conceptual del trastorno por déficit de atención e hiperactividad. Revista de Especialidades Médico-Quirúrgicas, 16, 39-44.

*Toplak, M. E., Jain, U., \& Tannock, R. (2005). Executive and motivational processes in adolescents with attention-defi- cit-hyperactivity disorder (ADHD). Behavioral and Brain Functions, 1, 8. doi: 10.1186/1744-9081-1-8.

Trujillo-Orrego, N., Ibáñez, A., y Pineda, D. E. (2012). Validez del diagnóstico de trastorno por déficit de atención/hiperactividad: de lo fenomenológico a lo neurobiológico. Revista de Neurología, 54, 367-379.

Van Duijvenvoorde, A. C. K., Bredman, J., \& Huizenga, H. (2012). Age-related changes in decision making: Comparing informed and non informed situations. Developmental Psychology, 48, 192-203. doi: 10.1037/a0025601.

Verdejo-García, A., y Tirapu-Ustárroz, J. (2012). Neuropsicología clínica en perspectiva: retos futuros basados en desarrollos presentes. Revista de Neurología, 54, 180-186.

White, J. W., \& Buehler, C. (2012). Adolescent sexual victimization, ADHD symptoms, and risky sexual behavior. Journal of Family Violence, 27, 123-132. doi: 10.1007/s10896-0129411-y.

Wilbertz, G., Trueg, A., Sonuga-Barke, E. J., Blechert, J., Philipsen, A., \& Tebartz van Elst, L. (2013). Neural and psychophysiological markers of delay aversion in attention-deficit hyperactivity disorder. Journal of Abnormal Psychology, 122, 566-572. doi: 10.1037/a0031924.

Willcutt, E. G. (2012). The prevalence of DSM-IV attention-deficit/hyperactivity disorder: a meta-analytic review. Neurotherapeutics, 9, 490-499. doi: 10.1007/s13311-012-0135-8.

Wolraich, M. L., Wibbelsman, C. J., Brown, T. E., Evans, S. W., Gotlieb, E. M., Knight, J. R., ... Wilens, T. (2005). Attention-deficit/hyperactivity disorder among adolescents: A review of the diagnosis, treatment, and clinical implications. Pediatrics, 115, 1734-1746.

Yasumura, A., Takimoto, Y., Nakazawa, E., \& Inagaki, M. (2016). Decision Making in Children with Attention-Deficit/ Hyperactivity Disorder. Open Journal of Pediatrics, 6, 158. doi: 10.4236/ojped.2016.62023.

Ziegler, S., Pedersen, M. L., Mowinckel, A. M., \& Biele, G. (2016). Modelling ADHD: a review of ADHD theories through their predictions for computational models of decision-making and reinforcement learning. Neuroscience \& Biobehavioral Reviews, 71, 633-656. doi: 10.1016/j.neubiorev.2016.09.002. 
